\title{
Transatlantica
}

Revue d'études américaines. American Studies Journal

$2 \mid 2011$

Sport et société / Animals and the American

Imagination

\section{Henri Cartier-Bresson / Paul Strand, Mexique}

\section{2-1934}

Exposition à la Fondation Henri Cartier-Bresson, du 11 janvier au 22 avril 2012. L'exposition sera présentée du 13 mai au 2 septembre au Point du Jour Centre d'art à Cherbourg.

\section{Didier Aubert}

\section{(2) OpenEdition}

\section{Journals}

Édition électronique

URL : https://journals.openedition.org/transatlantica/5594

DOI : $10.4000 /$ transatlantica.5594

ISSN : 1765-2766

Éditeur

Association française d'Etudes Américaines (AFEA)

Référence électronique

Didier Aubert, « Henri Cartier-Bresson / Paul Strand, Mexique 1932-1934 », Transatlantica [En ligne], 2 I 2011, mis en ligne le 05 juin 2012, consulté le 01 février 2023. URL : http://journals.openedition.org/ transatlantica/5594; DOI : https://doi.org/10.4000/transatlantica.5594

Ce document a été généré automatiquement le 1 février 2023.

\section{cc)}

Creative Commons - Attribution - Pas d'Utilisation Commerciale - Pas de Modification 4.0 International - CC BY-NC-ND 4.0

https://creativecommons.org/licenses/by-nc-nd/4.0/ 


\section{Henri Cartier-Bresson / Paul Strand, Mexique 1932-1934}

Exposition à la Fondation Henri Cartier-Bresson, du 11 janvier au 22 avril 2012. L'exposition sera présentée du 13 mai au 2 septembre au Point du Jour Centre d'art à Cherbourg.

\section{Didier Aubert}

1 Après un détour par les origines de la photographie sociale américaine lors d'une récente rétrospective Lewis Hine, la Fondation Cartier-Bresson revient vers l'œuvre du grand photographe français à qui elle doit son nom. Elle ne quitte pas pour autant les Amériques. Par le biais de cette nouvelle exposition, l'institution parcourt à nouveau l'espace transatlantique qui façonne pour partie ce que nous savons du canon de la photographie des années 30. Le visiteur retrouve ici, en effet, le triangle New YorkParis-Mexico qui était déjà présent, il y a quelques années, dans Documentary and antigraphic photographs by Cartier Bresson, Walker Evans and Alvarez-Bravo. A l'époque, il s'agissait de recréer l'exposition ayant réunit ces trois grands documentaristes à la galerie Julien Levy, à New York, en 1935. Avec Henri Cartier-Bresson / Paul Strand, Mexique 1932-1934, le rapprochement apparait plus ténu. Le visiteur se voit proposer un rapprochement entre Cartier-Bresson et l'un de ses illustres contemporains, le photographe et cinéaste américain Paul Strand (1890-1976), disciple d'Alfred Stieglitz et de Lewis Hine, double héritier de la photographie d'art et du documentaire social, et qui termina sa vie en France après avoir fui le maccarthysme. Si ces photographies de Cartier-Bresson sont déjà familières (la plupart sont tirées de son fameux Scrapbook, objet d'une exposition antérieure), la présentation du travail de Strand, bien moins connu en France, est en soi un petit événement.

2 L'exposition prend prétexte d'un séjour effectué par Cartier-Bresson au Mexique en 1934, au moment même où le New Yorkais Paul Strand travaille pour le gouvernement mexicain, deux ans après son arrivée dans le pays. Le parcours des deux hommes ne se croisera réellement qu'un peu plus tard aux États-Unis, lorsqu'ils se retrouveront côteà-côte au sein du collectif documentaire Nykino (plus tard mieux connu sous le nom de Frontier Film). Ils ne sont pas tout à fait de la même génération, et la différence de style 
entre les images proposées ici est assez frappante. Mais le Mexique - au même titre que l'Espagne de la Guerre Civile - est un espace fertile pour la photographie des années 30. C'est en partie ce qui fait la force, et sans doute certaines limites, de l'exposition, car on y devine, plus qu'on ne le comprend, l'attrait singulier de ce pays d'images dans les premières décennies $\mathrm{du} 20^{\mathrm{e}}$ siècle ${ }^{1}$.

3 Il y a quelques mois seulement, se tenait à l' Université de Princeton une conférence intitulée Did modern photography begin in Mexico ? L'un des attraits de cette exposition est précisément de nous rappeler ce chapitre «mexicain » où se rejoignent le modernisme et le documentaire photographiques. A partir des années 20, on croise en effet, à Mexico et ailleurs, des figures aussi marquantes que les Américains Edward et Brett Weston, l'Italienne Tina Modotti ou le Mexicain Manuel Alvarez-Bravo. Le travail de Paul Strand se voit consacrer une exposition dès 1933, alors même que la Fondation Guggenheim vient de lui refuser une bourse. Une coupure de presse exhumée des archives de Cartier-Bresson rend compte d'une autre exposition organisée quelques mois plus tard au Palais des Beaux Arts de Mexico : ses photographies y sont comparées à celles d'Alvarez Bravo. L'auteur de cette critique n'est autre que l'écrivain Langston Hughes, lui-même marqué par ses très nombreux séjours dans le pays où vivait son père.

4 En exposant les images réalisées par les deux hommes dans deux salles différentes, les commissaires de l'exposition prennent soin de ne pas recréer artificiellement une communauté de vue qui n'existait pas au moment où ils travaillaient pourtant si près l'un de l'autre. Ils semblent toutefois vouloir suggérer que Strand et Cartier-Bresson vécurent au même moment, et dans le même pays, une étape importante de leur carrière de photographes "engagés ». Pourtant, si Clément Chéroux évoque à juste titre un "tropisme politique", dans le catalogue de l'exposition, pour qualifier l'attirance des deux hommes pour le pays de Diego Rivera et de la première révolution marxiste du $20^{\mathrm{e}}$ siècle, l'exposition ne présente que quelques documents suggérant de manière explicite cette dimension de leur travail. Un article de Life publié en mai 1937 vante les qualités esthétiques de Las Redes (The Wave, 1936), documentaire réalisé par Strand pour le Ministère de l'éducation mexicaine, tout en en déplorant ses lourdeurs didactiques. Dans la présentation des images de Cartier-Bresson, en revanche, cette mise en perspective sociale et politique est quasiment absente. Les circonstances même de la venue du Français, à l'occasion d'une expédition ethnographique finalement avortée, auraient peut-être mérité quelques explications. Suivant la politique habituelle de la Fondation, c'est pourtant au travers d'un parcours à travers des images très peu commentées ou contextualisées que nous sommes invités à pénétrer le regard de deux artistes emblématiques de la " grande histoire » de la photo.

\section{Le ciel et la rue}

Héritiers malgré eux d'une tradition "pittoresque» du regard photographique européen ou américain sur la société mexicaine, les deux hommes arrivent dans un Mexique où le nouveau Parti Révolutionnaire Institutionnel prétend pérenniser les acquis de la Révolution de 1910 à travers la puissance d'un appareil d'État dirigé par un parti hégémonique. Comme dans beaucoup d'autres pays à l'époque, le Mexique exalte la grandeur nationale à travers un certain discours populiste et une politique culturelle volontariste. Employé par le gouvernement, Strand produit des photographies qui 
semblent reprendre les clichés d'une mexicanité rurale et « indienne » en vogue dans les discours officiels ${ }^{2}$. Pourtant, sa fascination pour l'art religieux doit être perçue dans le contexte des conflits brutaux et incessants entre le pouvoir politique issu de la révolution et une paysannerie profondément attachée au Catholicisme. James Krippner prétend que Strand avait sans doute l'ambition de dresser une histoire sociale visuelle du Mexique, notamment à travers les 20 tirages rassemblés dans l'ouvrage Photographs of Mexico, publié en $1940^{3}$. L'exposition aurait sans doute gagné à évoquer ces pistes d'interprétations, mais elle offre au moins l'inestimable plaisir de contempler dans leur ensemble ces 20 photographies, regroupées pour offrir un tableau très frappant de la vision de Strand. La cohérence de ce véritable photo essay (et de la totalité des images de Strand proposées ici) se tisse dans les correspondances subtiles qui renvoient des visages de paysans à ceux des statues du Christ, empreints de gravité et de douleur. Loin des États-Unis en proie à la crise, Strand semble trouver ici une forme de transcendance, entre les portails blancs des églises et les reflets presque noirs du ciel.

Il est d'autant plus frappant de constater que Cartier-Bresson ne lève jamais le regard vers les cieux, et se soucie peu des églises. Nous sommes ici dans un genre particulier de street photography qui hésite entre poésie du quotidien et conscience sociale. Le Français arpente les trottoirs le regard souvent baissé, pour photographier ceux qui y vivent, assis ou couchés: vendeuses de journaux, vagabonds, enfants... Un homme agenouillé, la tête contre le pavé, semble prier; il est sans doute en train de finir de cuver la bière ingurgitée la veille. Les paysans de Strand ont gagné la grande ville, sans y gagner au change. Pourtant, les sourires éclatants de ces petites gens est le bien le mieux partagé, que ce soit sur le visage d'une enfant nue, d'une prostituée attendant le client ou d'un vendeur de cercueils. Les seules sculptures qui intéressent vraiment Cartier-Bresson sont celles qui se construisent dans l'instant, et à travers l'objectif, la juxtaposition inattendue de deux visages derrière une grille, d'une énorme outre en peau de porc et de deux poteries évoquant des attributs sexuels gigantesques. Si la mort et la pauvreté sont souvent présentes, la sexualité et la vie débordent de presque chaque image.

7 On peine dès lors à résister aux généralisations tentantes. L'austérité « puritaine » qui marque une partie de la photographie moderniste américaine - et dont Strand explorera les origines dans un ouvrage sur la Nouvelle Angleterre publié en $1950^{4}-$ ne saurait pourtant à elle seule expliquer la différence de style entre le New-yorkais et le Français, largement inspiré par le Surréalisme. Le rapprochement de ces deux séries d'images fournit surtout un nouvel exemple des liens étroits entre aspirations modernistes et souci documentaire dans la photographie des années 30, une problématique particulièrement en vogue dans l'historiographie récente ${ }^{5}$. Que cet «âge d'or" photographique ait cherché quelques réponses à cette question du côté du Mexique, rien que de très normal finalement. 


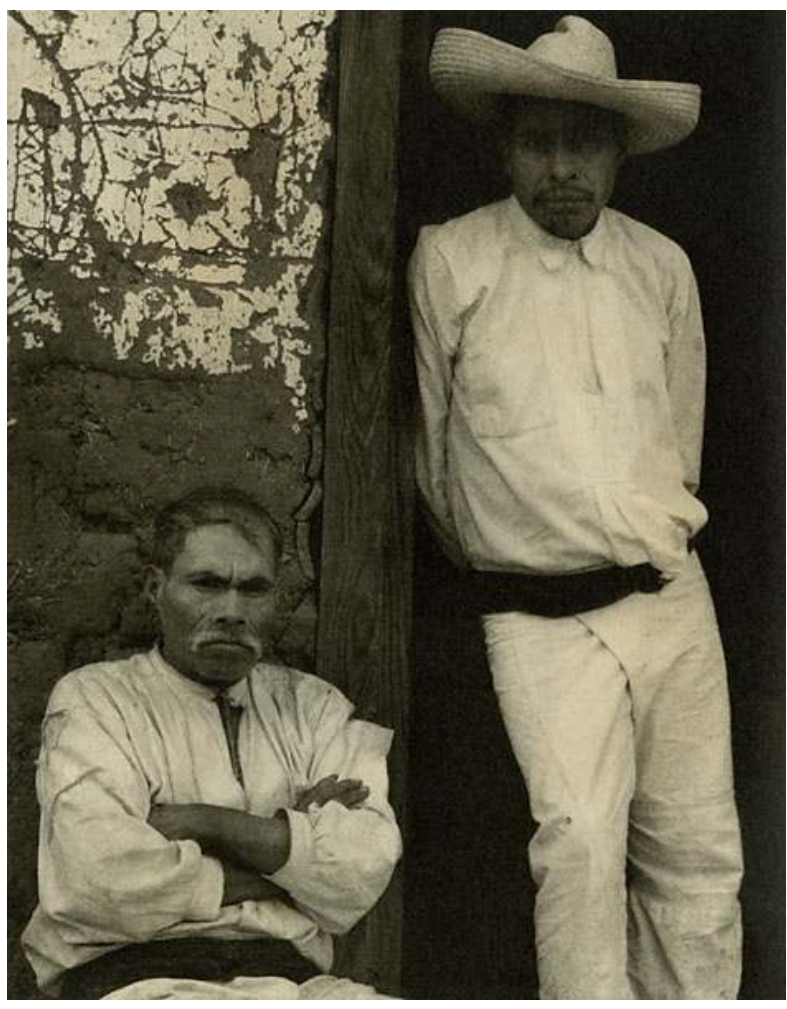

Paul Strand, Men of Santa Ana, Lake Patzcuaro, Michoacan, 1933. Reproduit avec l'aimable autorisation de la Fondation Henri Cartier-Bresson

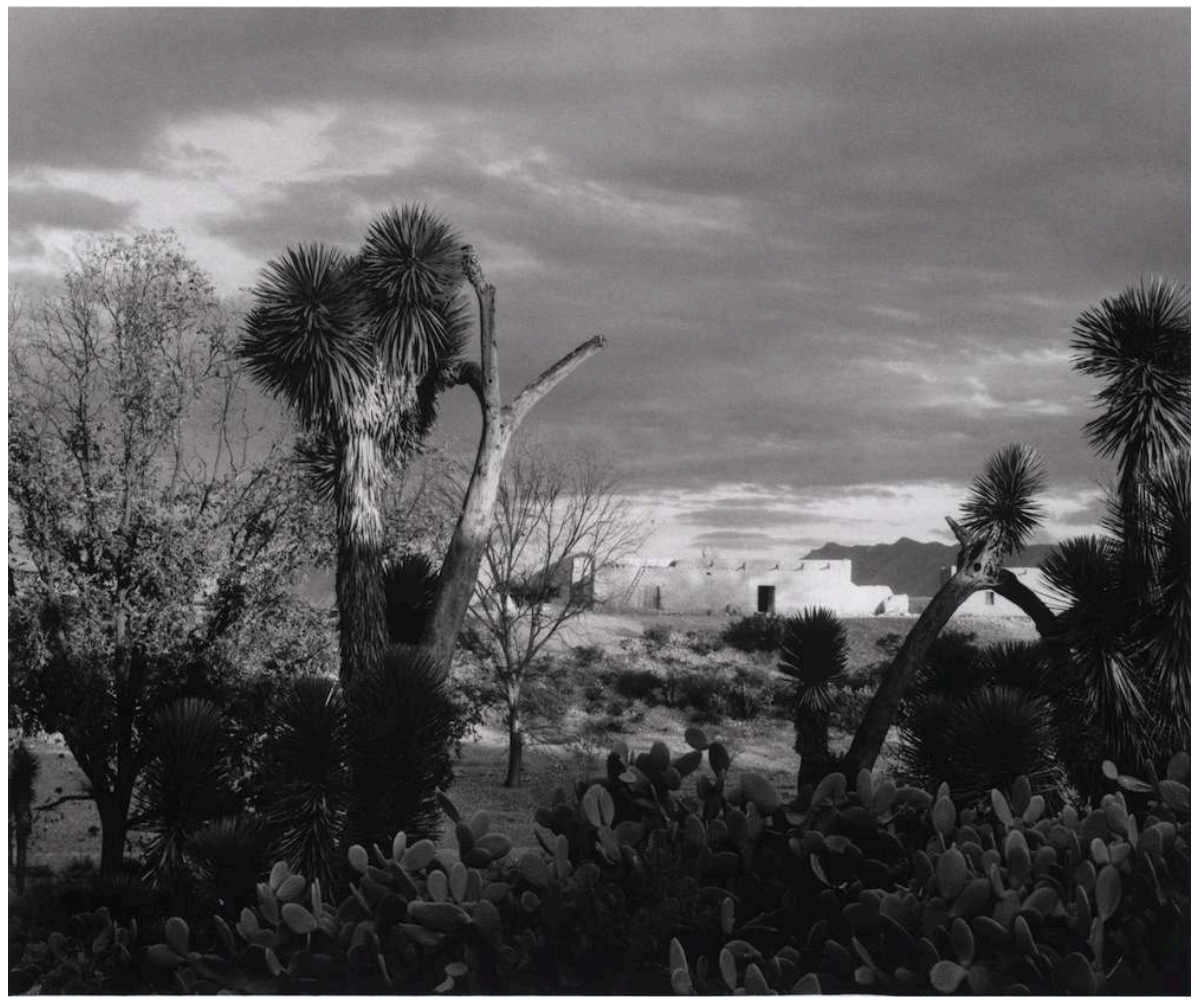

Paul Strand, Landscapenear Saltillo, 1932.Reproduit avec l'aimable autorisation de la Fondation Henri Cartier-Bresson. 


\section{NOTES}

1. Voire bien avant, comme le montre Serge Gruzinski, La guerre des images de Christophe Colomb à «Blade Runner» (1492-2019), Paris, Fayard, 1990.

2. Voir John Mraz, Looking for Mexico: Modern Visual Culture and National Identity (chapitre 2), Durham, North Carolina, Duke University Press, 2009.

3. James Krippner, Paul Strand in Mexico, New York, Aperture, 2010.

4. Nancy Newhall et Paul Strand, Time in New England: Photographs by Paul Strand,New York, Aperture, 1950. On se souvient aussi de l'utilisation par Lincoln Kirstein de l'adjectif «puritanical» pour décrire le travail de Walker Evans dans la préface d'American Photographs, New York, Museum of Modern Art, 1938.

5. Voir par exemple Jeff Allred, American Modernism and Depression Documentary, Oxford, Oxford University Press, 2010; Sharon Corwin et al., American Modern: Documentary Photography by Abbott, Evans and Bourke-White, Berkeley, University of California Press, 2010; Esther Gabara, Errant Modernism : The Ethos of Photography in Mexico and Brazil, Duke University Press, 2008.

\section{INDEX}

Thèmes : Trans'Arts 